\title{
PROTOPLASMIC CONTRACTIONS RESEMBLING PLAS- MOLYSIS WHICH ARE CAUSED BY PURE DISTILLED WATER
}

\author{
W. J. V. OSTERHOUT \\ (WITH SIX FIGURES)
}

True plasmolysis is produced only by solutions which are hypertonic, but appearances almost or quite indistinguishable from it may be brought about by hypotonic solutions. ${ }^{x}$ Some light is thrown on the nature of this result by a study of certain cases in which it is caused by pure distilled water. ${ }^{2}$

Material for such study is afforded by marine plants. The root tips of the eel-grass (Zostera marina) are well adapted to this purpose. The roots were carefully removed from the sand in which they were growing and immediately placed in sea water. Some of the younger roots (which had not yet become brown at the end) were selected. About an inch of the root tip was removed and placed on a hollow-ground slide in such a way that the young root hairs did not come into contact with the glass. The root tip was covered with sea water and examined without a cover glass (by means of an $8 \mathrm{~mm}$. objective and an ocular magnifying ten times). Root tips which were shown by such examination to be normal in appearance were fastened by means of vaseline to cover glasses which were then attached to irrigation chambers of the form shown in fig. I. Care was taken to prevent the young root hairs from touching the glass or the vaseline.

The arrangement of the apparatus may be understood from figs. I and 2. The irrigation chamber consists of an ordinary

${ }^{x}$ Cf. Bot. Gaz. 46:53. I908.

${ }^{2}$ Water twice distilled from glass is usually regarded as pure, especially when the first and last thirds of the distillate are rejected, but such water may be toxic to plants as long as any part of the apparatus is new. The water used in these experiments was prepared with due regard to these facts. In place of stoppers, plugs of absorbent cotton were used; contamination by spattering was prevented by baffling plates of glass and glass wool. The water so obtained was not toxic to such test objects as sensitive species of Spirogyra and the root hairs of Gypsophila.

Botanical Gazette, vol. 55] 
glass slide with a circular opening ( $5 \mathrm{~mm}$. in diameter) into which is fitted a glass cylinder $(c) \mathrm{I} 2 \mathrm{~mm}$. high upon which is cemented a glass disc $(d)$; surrounding this is another cylinder, the width of the space between the two being about I mm.; a part of this space is filled with paraffin (e). The outer cylinder is pierced on both sides by small glass tubes $(b, b)$. The outer cylinder projects above the inner so that when the cover glass $(f)$ is in place the width of the space between $(d)$ and $(f)$ is a little less than I mm. It is necessary to have this space narrow, since

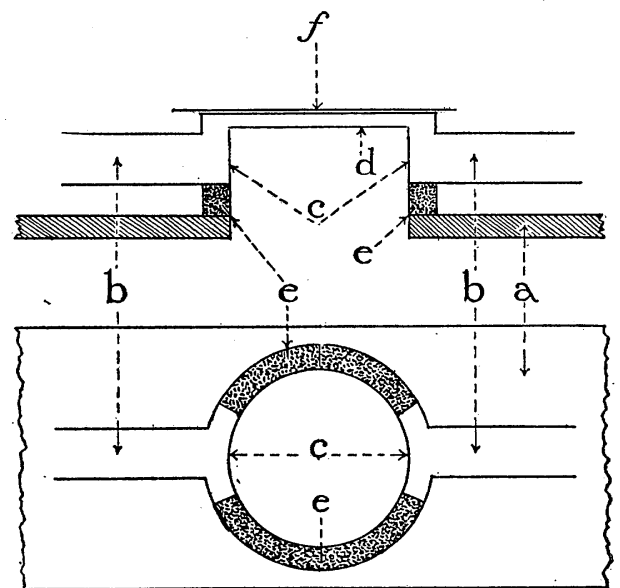

FIG. x.-Sectional views of irrigation chamber: $a$, slide; $b$, inlet and outlet tubes; $c$, glass cylinder; $d$, glass plate; $f$, cover glass (upper line) and material (lower line). otherwise when introducing a solution of greater specific gravity than the one with which the chamber is filled, the new solution may flow over the bottom of the space without coming into contact with the plants, which are fastened to the under side of the cover glass. The paraffin $(e)$ extends

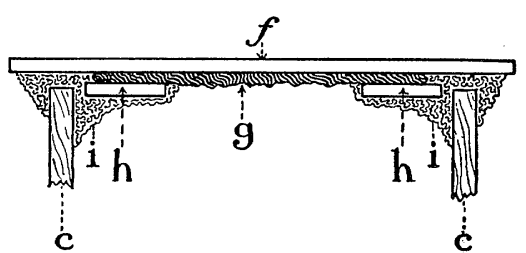

FIG. 2.-Material in irrigation chamber: $c$, glass cylinder; $f$, cover glass; $g$, material; $h$, bits of cover glass; $i$, vaseline. -Sectional view. downward to the slide; the liquid is in consequence obliged to pass upward through the space between $(d)$ and $(f)$ before it can flow out at the opposite side, and it must therefore bathe the plants which are attached to the cover glass $(f)$.

The attachment is made in the manner shown in fig. 2. The plant is first fastened to the cover glass $(f)$ by vaseline ( $i$, indicated by the dotted area) and a drop 
of sea water is placed on it; bits of cover glass $(h, h)$ are then thickly covered with vaseline, and pressed down upon it to hold it in place. The outer cylinder is smeared with vaseline and sea water is then poured into a funnel which is connected by a rubber tube with the inlet tube (b); as soon as all the air has been expelled from this tube and the chamber is so full of sea water that the surface of the liquid is decidedly convex, the cover glass (with the attached root tip) is inverted and pressed down upon the outer cylinder in the manner shown in the figure. Care should be taken during the subsequent irrigation not to admit air to the inlet tube.

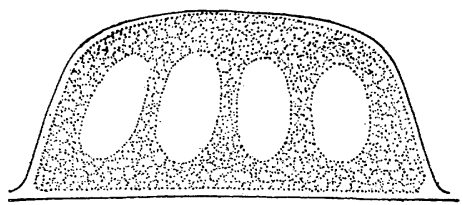

FIG. 3

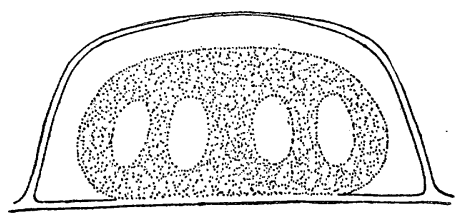

FIG. 5

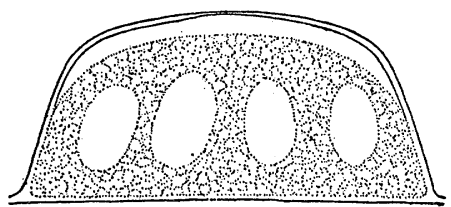

FIG. 4

FIGs. 3-5.-Optical section of young root hair cell of Zostera marina (diagrammatic); fig. 4, the cell shown in fig. 3 after treatment with distilled water (diagrammatic); fig. 5 , the cell shown in fig. 4 after more prolonged treatment with distilled water (diagrammatic).

After being placed in the chamber, the root tips were irrigated for a time with sea water while camera lucida sketches were made of root hairs in various stages of development. Each of the cells which had been sketched was than kept under observation during the subsequent irrigation with distilled water. Root tips which were irrigated with sea water throughout the experiment served as controls.

The application of distilled water causes a contraction of the protoplasm which often closely resembles the true plasmolysis produced by hypertonic sea water (which has been concentrated by evaporation) or by hypertonic sugar solutions. Figs. 4 and 5 show the appearance of such cells. The mode and the degree 
of contraction vary somewhat, but in general the variations in true plasmolysis are of the same sort as in what may be conveniently called the false plasmolysis. We may use the term "false plasmolysis" to designate not only the contraction produced by distilled water but also that which is caused by

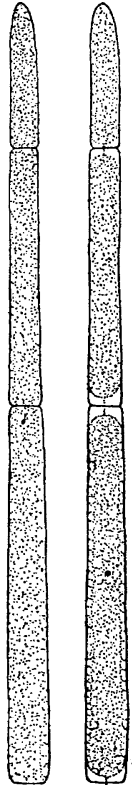

Fig. 6.-Surface view of the end of a hair of Polysiphonia violacea: at the left in natural condition; at the right after treatment for two minutes with distilled water (diagrammatic). hypotonic solutions.

The contraction may take place rather slowly, in many cases requiring half an hour or more to reach the stage shown in fig. 4. True plasmolysis may take place much more rapidly. But this distinction does not hold generally, for in many cases contractions which closely simulate true plasmolysis may take place with great rapidity. To give a single example of this, the behavior of the colorless terminal cells and of hairs of Polysiphonia violacea ${ }^{3}$ may be described. On being irrigated with distilled water these cells contract very rapidly, so that at the end of two minutes they reach the stage shown at the right in fig. 6 , in which they are practically indistinguishable from cells plasmolyzed by hypertonic sea water or hypertonic sugar solutions. The older cells reach the same stage more slowly and betray by the alteration of their chromatophores that they are undergoing false plasmolysis. It should be noted that by applying hypertonic solutions of many salts, both true and false plasmolysis may be produced simultaneously. These contractions are (as a rule) irreversible, at least as soon as they have passed a certain stage.

The effects which have been described as due to distilled water were also produced by water taken directly from ponds, rivers, and springs; they are not due, therefore, to toxic substances resulting from the process of distillation; this point is emphasized because attention has previously been drawn to the

3 Kindly identified by Dr. W. G. FarLow. 
fact that water distilled in a metal still produces such results in Spirogyra, while pure distilled water does not.

The cause of these effects lies in an increase in the permeability of the plasma membrane (and likewise of internal cell membranes), as the result of which some or all of the substances which maintain the osmotic pressure of the cell diffuse out; the protoplasm then shrinks as the result of loss of water from the vacuoles, which in consequence become smaller, as is shown in figs. 3-5. This is often followed by an apparent "coagulation" of the protoplasm, which is sometimes evidenced by the assumption of an irregular outline. Most of the characteristic features of cytolysis as described for animal cells are lacking. In some cases, however (particularly in cells which are not surrounded by a cell wall), they occur.

These effects might naturally be ascribed to the absorption of water by the protoplasm, but they cannot be due to this cause, for observation shows that the cells do not increase in size as they would if water were absorbed. In some cases a few of the cells burst when transferred to distilled water, but the majority do not burst or even swell noticeably. This is probably due in some cases to the fact that swelling is prevented by the cell wall, for some cells which lack the cell wall (for example, spores of Polysiphonia) may swell in distilled water. Moreover, it was found that isotonic solutions of cane sugar produce the same effects as distilled water although not as rapidly.

The increased permeability must be due, therefore, to the loss of certain substances upon which the maintenance of the normal permeability depends. The most important of these are undoubtedly the inorganic salts. If the concentration of salts be lowered beyond a certain point, the permeability of the membrane increases very rapidly. This is shown by experiments in which the increase of permeability is directly measured by electrical means. In sea water plus an equal amount of distilled water the cells do not shrink, but with the addition of three volumes of distilled water they may begin to shrink in seven hours or less, and with increasing amount of distilled water shrinkage takes place more and more rapidly. These remarks apply only to balanced solutions such as sea water. 
The effect of unbalanced solutions on permeability has been discussed elsewhere. ${ }^{4}$

The facts described above have an important bearing on certain theories recently advocated by some biologists and colloid chemists. According to these authors, the effects which are usually attributed to osmotic pressure are in reality due to imbibition or to the giving up of water by the protoplasm, without the intervention of a semipermeable membrane. It would not be possible on this theory to account for the shrinkage of the protoplasm of a cell to half its volume when transferred from sea water to distilled water, especially when the process is irreversible. But the explanation given above - the increase of permeability of a semipermeable membrane -not only agrees with the facts described here, but also with those derived from a variety of other material, and by the use of entirely different methods.

Laboratory of Plant Physiology

HARVARD UNIVERSITY

${ }_{4}$ Science, N.S. 35: I12. I9I2;36:350. I9I2. 